\title{
Glue drops inside hadrons
}

\author{
B.Z. Kopeliovich ${ }^{1}$, B. Povh ${ }^{2 \dagger}$ Ivan Schmidt $^{1}$ \\ ${ }^{1}$ Universidad Tecnica Federico Santa Maria, Valparaiso, Chile, and \\ Institut für Theoretische Physik der Universität, Heidelberg, Germany \\ ${ }^{2}$ Max-Planck-Institut für Kernphysik, 69029 Heidelberg, Germany
}

\begin{abstract}
We present experimental evidences for the existence of a semi-hard scale in light hadrons. This includes the suppression of gluon radiation that is seen in high mass hadron diffraction; the weak energy dependence of hadronic total cross sections; the small value of the Pomeron trajectory slope measured in photoproduction of $J / \Psi$; shortage of gluons in the proton revealed by an unusual behavior of the proton structure function in the soft limit. All these observations suggest that gluons in hadrons are located within spots of a small size relative to the confinement radius.
\end{abstract}

\section{Introduction}

There is growing theoretical and experimental support leading towards the existence of a nonperturbative scale smaller than the usual $1 / \Lambda_{Q C D} \sim 1 \mathrm{fm}$, and which is related to the gluonic degrees of freedom. First, an analysis of hadronic matrix elements of the gluonic contribution to the energy momentum tensor, using the QCD sum rules approach, gives a value of $0.3 \mathrm{fm}$ for the radius of the corresponding form factor [1]. From the lattice side, numerical simulations of the gluon two point correlation function turn out a value of also $0.2-0.3 \mathrm{fm}$ for the correlation length [2], and the energy of the QCD string appears concentrated in a tube of radius $0.3 \mathrm{fm}$ in the transverse direction [3]. On the other side, it has been shown that the instanton radius peaks approximately at $1 / 3 \mathrm{fm}$ [4]. Furthermore, high statistics data for diffractive gluon bremsstrahlung in hadronic collisions is difficult to explain unless gluons in the proton have transverse momenta as high as about $0.7 \mathrm{GeV}$ [5]. This has been confirmed by studies of diffractive parton distributions, which concluded that they have a rather small transverse size [6]. What actually happens is that the smallness of the gluon clouds slows down Gribov diffusion of the gluons in transverse plane, and this results in a small slope of the Pomeron trajectory in hard reactions [7], in agreement with data. More arguments in favor of small gluonic spots coming from DIS can be found in [8]. Some of these results have been corroborated by recent studies of the spatial distribution of gluons in the transverse direction at small $x$ [9]. Here we overview the available experimental evidences for the presence of a semihard scale in hadronic structure.

\footnotetext{
${ }^{\dagger}$ speaker
} 


\section{Gluon radiation is suppressed}

If gluons in hadrons are located within small spots of radius $r_{0}$, they have enlarged transverse momenta $q_{T} \sim 1 / r_{0}$. Such gluons cannot be resolved by soft interactions and be shaken off, which means that the bremsstrahlung cross section should be suppressed compared to perturbative estimates.

However, in the case of soft inelastic collisions followed by multiparticle production the events with or without gluon radiation look alike. In both cases the produced particles build a plateau in rapidity, and then it is difficult to find any definite signature of the radiated gluons.

Diffraction offers an exclusive possibility to identify gluon radiation. A high-energy hadron can dissociate diffractively either via excitation of the valence quark skeleton, or by radiating gluons. These two mechanisms are characterized by different dependence on the effective mass, $M_{X}$, of the excitation,

$$
\frac{d \sigma(h p \rightarrow X p)}{d M_{X}^{2}}= \begin{cases}\frac{1}{M_{X}^{3}} & \text { excitation of the quark skeleton } \\ \frac{1}{M_{X}^{2}} & \text { diffractive gluon bremsstrahlung }\end{cases}
$$

The $M_{X}$-dependence at large $M_{X}$ correlates with the spin of the slowest particle produced in the excitation. Only a vector particle, i.e. a gluon, can provide the $1 / M_{X}^{2}$ dependence.

Thus, one can single out the cross section of diffractive gluon radiation from the large mass tail of the $M_{X}$-distribution. An analysis [10] of diffractive data shows that gluon radiation is amazingly weak. In order to understand that one can interpret diffraction in terms of the Pomeron-proton total cross section, as is shown in Fig. 1. If we treat the Pomeron like a gluonic

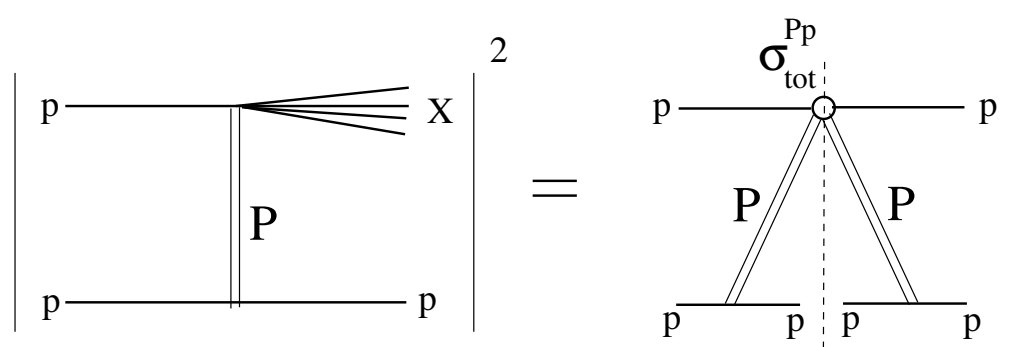

Fig. 1: The cross section of diffractive excitation of a proton expressed in terms of the total Pomeron-proton cross section.

dipole, one may expect a cross section $9 / 4$ times larger than for a $\bar{q} q$ dipole. Comparing with the pion-proton cross section, say $25 \mathrm{mb}$, one arrives at the estimate of about $50 \mathrm{mb}$. However, data suggest quite a smaller value, about $2 \mathrm{mb}$. A straightforward explanation for such a dramatic disagreement would be a much smaller size of the gluonic dipole (Pomeron) compared to the quark-antiquark dipole (pion). Thus, one concludes that gluons should be located within small spots in the proton.

Although it is not quite rigorous, one might try to estimate the diffractive radiation cross section perturbatively, and in this case the result exceeds data by more than an order of magnitude. To reduce the cross section down to the observed value one should assume that the mean quarkgluon separation is as small as $r_{0}=0.3 \mathrm{fm}$ [5]. With such a modified quark-gluon light-cone 
distribution function, the effective triple-Pomeron coupling has the form [5],

$$
\left.G_{3 \mathbf{P}}(0) \equiv\left(1-x_{F}\right)^{\alpha_{\mathbb{P}}(0)} \frac{d \sigma_{s d}(p p \rightarrow p X)}{d x_{F} d p_{T}^{2}}\right|_{p_{T}=0}=\frac{81 \alpha_{s} \sigma_{0}}{(16 \pi)^{2}} \ln \left[\frac{2\left(r_{0}^{2}+R_{0}^{2}\right)^{2}}{R_{0}^{2}\left(2 r_{0}^{2}+R_{0}^{2}\right)}\right] .
$$

Here we assume that $1 \gg 1-x_{F} \gg s_{0} / s$, where $s_{0} \sim 1 \mathrm{GeV}^{2}$. The energy dependent parameters $\sigma_{0}(s)$ and $R_{0}(s)$ controlling the shape of the universal dipole cross section [11] are defined in [5]. With $r_{0}=0.3 \mathrm{fm}$, the triple-Pomeron coupling eq. (2) agrees with the result of the triple-Regge analysis [10] of single diffractive data.

\section{Energy dependence of hadronic cross sections is weak}

It is well known that hadronic cross sections rise with energy approximately as $s^{\epsilon}$, where the exponent is quite small, $\epsilon \approx 0.1$. What is the origin of this small number? We do not expect any small parameters in the soft regime of strong interactions.

This problem is closely related to the topic of the previous section. In fact, the energy dependence is driven by gluon radiation which turns out to be suppressed. Before we saw a manifestation of this effect in diffraction, now in the total inelastic cross section.

Without gluon radiation the geometric cross section of two hadrons would be constant, since their transverse size is Lorentz invariant, i.e. is energy independent. The phase space for one gluon radiation is proportional to $\ln (s)$, so multigluon radiation leads to powers of $\ln (s)$ in the cross section. The calculations performed in [12] confirm this. The hadronic cross section was found to have the following structure,

$$
\sigma_{t o t}=\sigma_{0}+\sigma_{1}\left(\frac{s}{s_{0}}\right)^{\Delta}
$$

where $\sigma_{0}$ is the energy independent term related to hadronic collisions without gluon radiation. The second term in (3) is the contribution of gluon bremsstrahlung to the total cross section. Here the parameter $\sigma_{1}$ is expected to be small due to the smallness of the gluonic spots. Indeed, it was found in [12] that $\sigma_{1}=27 C r_{0}^{2} / 4$, where factor $C \approx 2.4$ is related to the behavior of the dipoleproton cross section, calculated in Born approximation at small separations, $\sigma\left(r_{T}\right)=C r_{T}^{2}$ at $r_{T} \rightarrow 0$.

The energy dependence of the second term in (3) was found to be rather steep, $\Delta=$ $4 \alpha_{s} / 3 \pi=0.17$. This exponent seems to be too large compared to the experimentally measured $\epsilon \approx 0.1$. There is, however, no contradiction due to the presence of the large energy independent term in (3). Approximating the cross section (3) by a simple power dependence on energy, the effective exponent reads,

$$
\epsilon=\frac{\Delta}{1+\sigma_{0} / \sigma_{1}\left(s / s_{0}\right)^{-\Delta}}
$$

So, one should expect a growing steepness of the energy dependence for the total cross section. One can estimate the value of $r_{0}$ demanding the effective exponent to be $\epsilon \approx 0.1$ in the energy range of fixed target experiments, say at $s \sim 1000 \mathrm{GeV}^{2}$. With $\sigma_{0}=40 \mathrm{mb}$ found in [12] one gets $r_{0}=0.3 \mathrm{fm}$. 
Thus, the observed slow rise of the total hadronic cross sections provides another evidence for the existence of small gluonic spots with transverse size $r_{0} \sim 0.3 \mathrm{fm}$.

One may expect a steeper energy dependence for heavier flavors. Indeed, for $J / \Psi$-proton scattering $\sigma_{0}$ is so small, that $\epsilon \approx \Delta$. Indeed, data for $J / \Psi$ photoproduction from HERA [13] show that $\epsilon \approx 0.2$. One should be careful, however, interpreting the data within the vector dominance model [14], and remember that Eq. (3) was derived assuming that $r_{0}$ is much smaller than the hadronic size, otherwise interferences should be included.

\section{Diffractive cone shrinks with energy slowly}

The prediction of a shrinkage of the diffraction cone has been one of the first achievements of the Regge theory. Indeed, data show that the elastic slope in hadronic collisions rises with energy as, $B_{e l}(s)=B_{0}+2 \alpha_{\mathbf{I P}}^{\prime} \ln \left(s / s_{0}\right)$, where $\alpha_{\mathbf{I P}}^{\prime} \approx 0.25 \mathrm{GeV}^{-2}$. This is about four times smaller than in binary processes mediated by other Reggeons, $\alpha_{\mathbf{R}}^{\prime} \approx 1 \mathrm{GeV}^{-2}$. Why?

The diffractive cone shrinkage is usually related to Gribov diffusion of gluons in the transverse plane. If each "step" in impact parameters, $\Delta b^{2}=r_{0}^{2}$, is small, the diffusion should proceed slowly. Indeed, a rather small value of the Pomeron trajectory slope was predicted in [12],

$$
\alpha_{\mathbf{I P}}^{\prime}=\frac{1}{2} \frac{d B_{e l}}{d \ln \left(s / s_{0}\right)}=\frac{\alpha_{s}}{3 \pi} r_{0}^{2}=0.1 \mathrm{GeV}^{-2} .
$$

This seems to be too small, in strong contradiction with value $0.25 \mathrm{GeV}^{-2}$ known from data for the elastic slope. One may wonder, why the same model [12] which predicts (5) describes well data for elastic slope, as is demonstrated in Fig. 2. The relatively large value of $\alpha_{\mathbf{I P}}^{\prime}$, turns out to result from unitarity saturation. Indeed, the elastic differential cross section (actually the amplitude) Fourier transformed to impact parameter representation (see details in [12]) demonstrate unitarity saturation at small impact parameters. In spite of the observed rise of the total cross section with energy, there is no room for further growth at small impact parameters, only the amplitudes of peripheral collisions rise with energy. This leads to a rising with energy radius of interaction directly related to the elastic slope. Thus, a substantial part of the observed energy dependence of the elastic slope and of the effective $\alpha_{e f f}^{\prime}=0.25 \mathrm{GeV}^{-2}$ is related to saturation of the unitarity bound. How to disentangle the two effects?

To get rid of unitarity corrections one can consider the interaction of a small dipole with a proton. For example photoproduction of a heavy quarkonium [7], or high $Q^{2}$ electroproduction of $\rho$. Then, the elastic amplitude is too small to be affected by unitarity (absorptive) corrections, and the energy dependence of the slope must be solely due to the rise of the gluon clouds, i.e. Gribov diffusion. This expectation of [12] was nicely confirmed in elastic photoproduction $\gamma+p \rightarrow$ $J / \Psi+p$ measured by the ZEUS experiment [13] which found $\alpha_{\mathbb{P}}^{\prime}=0.115 \pm 0.018 \mathrm{GeV}^{-2}$. The data and fit are depicted in Fig. 3

\section{There is a shortage of gluons at low scale}

As far as gluons are located within small spots, it is difficult to resolve them at low scale, $Q^{2}<$ $4 / r_{0}^{2}$. With poor resolution the proton looks like a 3-quark system containing no gluons. At the same time, no changes happen at higher $Q^{2}$ which resolve distances much smaller than the 


\section{ZEUS}

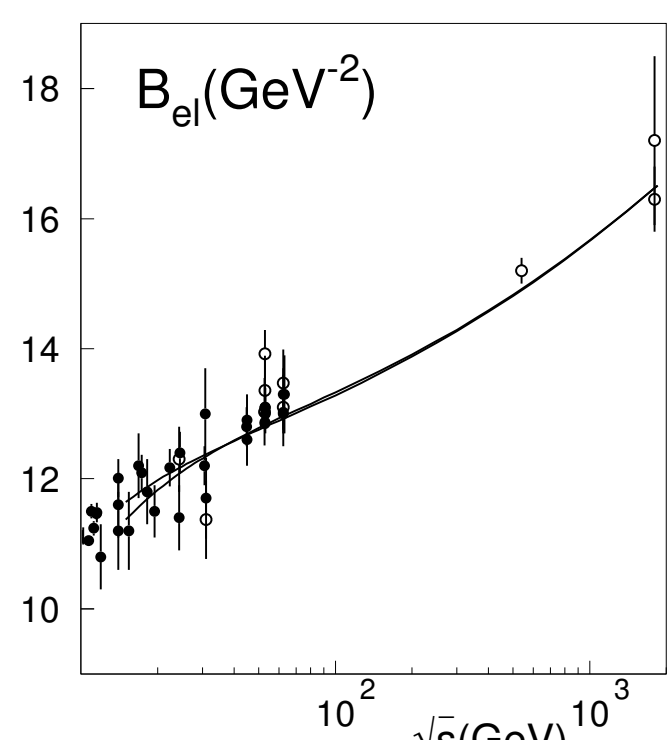

Fig. 2: Elastic slope for $p p / \bar{p} p$ (bottom/upper curves) collisions, calculated in [12] with the small Pomeron slope according to (5). The references to the data can be found in [12].

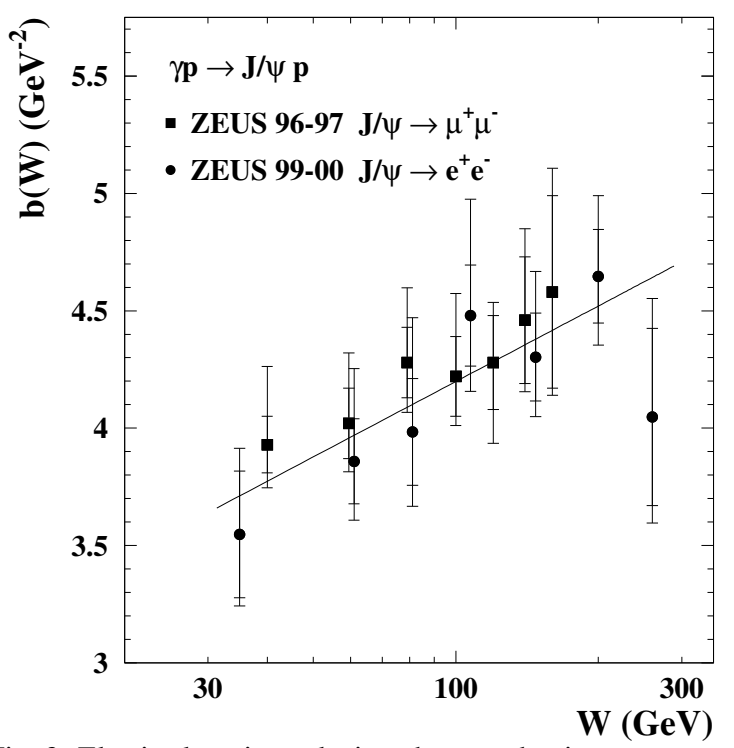

Fig. 3: Elastic slope in exclusive photoproduction $\gamma+p \rightarrow$ $J / \Psi+p$. Data are from the ZEUS experiment [13]. The solid curve is a fit with $\alpha_{\mathbf{I P}}^{\prime}=0.115 \pm 0.018 \mathrm{GeV}^{-2}$, in good agreement with (5).

size of gluonic spots. In this regime the gluon density is rising toward smaller $x$ unless related values of $Q^{2}$ become too low to resolve the spots. This explains the ZEUS data [15] depicted in Fig. 4 for logarithmic $Q^{2}$ derivative of the structure function. This derivative suddenly drops at $Q^{2}$ below few $\mathrm{GeV}^{2}$ showing that parton distributions are frozen below this scale, no evolution happens due to lack of gluons.

Summarizing, a comprehensive list of experimental evidences for existence of two scales in the hadronic structure is currently available. Many of them directly indicate a small gluonic spot structure of light hadrons.

The full size paper on the "Two Scales of Hadron Structure" is to be found in arXiv.org/abs/0708.3636.

Acknowledgments: We are thankful to Hans-Jürgen Pirner for helpful discussions. This work was supported in part by Fondecyt (Chile) grants numbers 1030355 and 1050519, and by DFG (Germany) grant PI182/3-1.

\section{References}

[1] V.M. Braun, P. Górnicki, L. Mankiewicz, Phys. Lett. B302 (1993) 291.

[2] M. D’Elia, A. Di Giacomo, Phys. Lett. B408 (1997) 315.

[3] G.S. Bali, Int. Conference in Quark Confinement and Hadorn Spectrum, Newport News (Virginia, USA), June 1998. 


\section{ZEUS 1995}

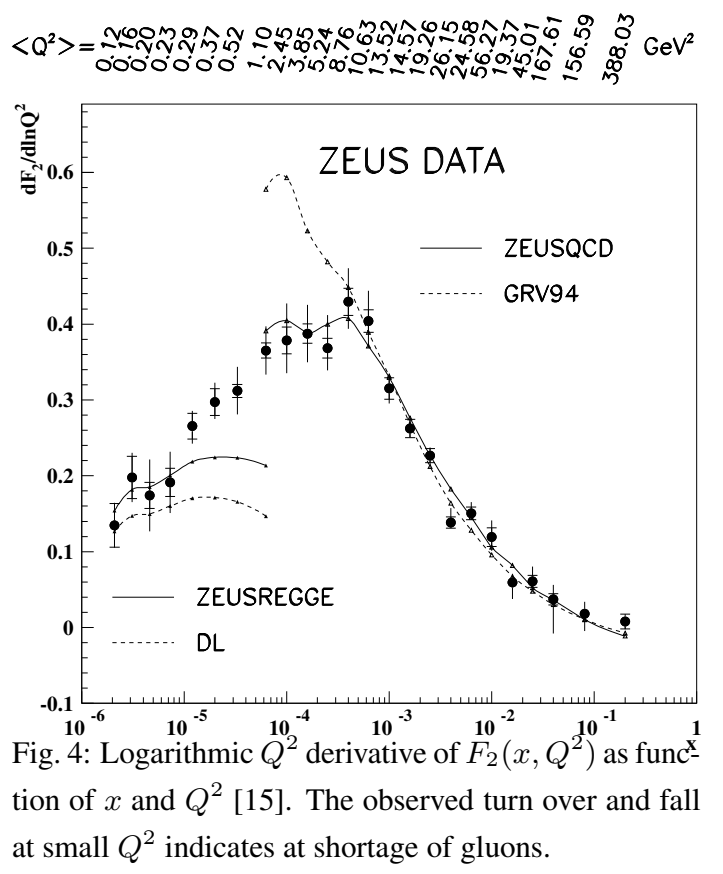

[4] E.V. Shuryak, Phys. Lett. B486 (2000) 378.

[5] B.Z. Kopeliovich, A. Schäfer, and A.V. Tarasov Phys. Rev. D62 (2000) 054022.

[6] F. Hautmann, D.E. Soper, Phys. Rev. D63 (2000) 011501.

[7] B.Z. Kopeliovich and B. Povh, J. Phys. G30 (2004) S999.

[8] E. Shuryak and I. Zahed, Phys. Rev. D69 (2004) 014011.

[9] M.I. Strikman and C. Weiss, Phys. Rev. D69 (2004) 054012.

[10] Yu.M. Kazarinov, B.Z. Kopeliovich, L.I. Lapidus, and I.K. Potashnikova, Sov. Phys. JETP 70 (1976) 1152.

[11] B.Z. Kopeliovich, L.I. Lapidus, and A.B. Zamolodchikov, Sov. Phys. JETP Lett. 33, 595 (1981); Pisma v Zh. Exper. Teor. Fiz. 33, 612 (1981).

[12] B.Z. Kopeliovich, I.K. Potashnikova, B. Povh, and E. Predazzi, Phys. Rev. Lett. 85 (2000) 507; Phys. Rev. D63 (2001) 054001.

[13] ZEUS Collaboration, S. Chekanov et al., Eur. Phys. J. C24 (2002) 345.

[14] J. Hüfner and B.Z. Kopeliovich, Phys. Lett. B426 (1998) 154.

[15] ZEUS Collaboration, J. Breitweg et al., Eur. Phys. J. C7 (1999) 609. 